\title{
Comparative study of the effectiveness of three learning environments: Hyper-realistic virtual simulations, traditional schematic simulations and traditional laboratory
}

\author{
Guadalupe Martínez, Francisco L. Naranjo, Ángel L. Pérez, and Maria Isabel Suero \\ Department of Physics, University of Extremadura, Avenida de Elvas, s/n, 06071 Badajoz, Spain \\ Pedro J. Pardo \\ Department of Computer and Network Systems Engineering, University of Extremadura, \\ C/Santa Teresa de Jornet, 38, 06800 Mérida, Spain
}

(Received 14 October 2010; published 31 October 2011)

\begin{abstract}
This study compared the educational effects of computer simulations developed in a hyper-realistic virtual environment with the educational effects of either traditional schematic simulations or a traditional optics laboratory. The virtual environment was constructed on the basis of JAVA applets complemented with a photorealistic visual output. This new virtual environment concept, which we call hyper-realistic, transcends basic schematic simulation; it provides the user with a more realistic perception of a physical phenomenon being simulated. We compared the learning achievements of three equivalent, homogeneous groups of undergraduates - an experimental group who used only the hyper-realistic virtual laboratory, a first control group who used a schematic simulation, and a second control group who used the traditional laboratory. The three groups received the same theoretical preparation and carried out equivalent practicals in their respective learning environments. The topic chosen for the experiment was optical aberrations. An analysis of variance applied to the data of the study demonstrated a statistically significant difference $(p$ value $<0.05$ ) between the three groups. The learning achievements attained by the group using the hyper-realistic virtual environment were 6.1 percentage points higher than those for the group using the traditional schematic simulations and 9.5 percentage points higher than those for the group using the traditional laboratory.
\end{abstract}

DOI: 10.1103/PhysRevSTPER.7.020111

PACS numbers: 01.40.Fk, 01.50.Lc

\section{INTRODUCTION}

\section{A. Previous studies of simulations in physics}

New information and communications technologies provide highly effective teaching tools based on the use of virtual platforms. In e-learning, diverse virtual teaching materials are used and students have found these materials to be very useful in facilitating their construction of knowledge [1,2]. According to Halloun [3], the use of increasingly sophisticated software promotes a constructivist approach to learning. Among these tools for physics teaching, computer simulations are particularly interesting [4-14]. Their use fosters conceptual development and change and helps students to comprehend many physical phenomena in different areas of study, such as mechanics $[15,16]$, optics [17-19], or even the entire science curriculum [20-22].

Before simulations can be integrated into the curriculum, however, their effectiveness needs to be evaluated. In recent years, the positive impact of computer simulations at different stages of the teaching and learning process has

Published by the American Physical Society under the terms of the Creative Commons Attribution 3.0 License. Further distribution of this work must maintain attribution to the author(s) and the published article's title, journal citation, and DOI. been documented. Snir, Smith, and Grosslight [23] state that computer simulations create a learning process that is far more efficient and applicable to real-world problems or situations. Other studies have demonstrated the way in which simulations benefit instructional approaches and capabilities and promote the development of general and, in particular, cognitive and metacognitive skills and conceptual understanding [12,15,16,20,21,24-29]. While many researchers have found that groups of pupils who have worked with computer simulations make greater strides in learning [12,30-32], others [33-35] have found that the benefits of learning through simulations are ambiguous. Some researchers have even suggested that an actual experiment will inevitably be more complex than a virtual one, so an attempt should always be made to observe a physical phenomenon in nature. Aleksandrova [36] believes that practical classes carried out in virtual environments can serve as an educational supplement but cannot replace the real laboratory.

\section{B. Drawback of traditional simulations}

Our own research group has had long-term experience (over 25 years) in the development and use of teaching materials and computer simulations [37-42]. In spite of the fact that the teaching effectiveness of these simulations has been repeatedly proven, in developing effective 
simulations we constantly face the challenge of how to adequately reproduce the phenomenon being studied. For example, experiments in virtual spaces provide a new way to present abstract concepts using realistic models. We have often seen that, in the more applied areas of science, such as optics, some students have serious difficulties in properly connecting the observations they make in the simulated model with what they see in reality. In purely theoretical areas, however, abstract constructions, such as those provided by traditional computer simulations, are sufficient. Therefore, in applied areas, it is important to include in the experiment not only the characteristic properties of the basic phenomenon but also a certain degree of reality. That is, the design and the degree of realism displayed in the simulation interface should match the purpose of the teaching.

Previous authors have analyzed the benefits and drawbacks of abstract versus idealized and concrete versus real models. Goldstone and Son [43], for instance, discuss the advantages of more concrete simulations as compared with simulations that use idealized models. They argue that the more concrete computer simulations are more helpful during the initial stages of learning but inhibit the students' ability to transfer the underlying abstract principles to new situations. According to DiFonzo et al. [44], a high level of detail in realistic representations of objects within the simulation can benefit students in their study of a given phenomenon by increasing the similarity between the simulation and the real world. Indeed, most virtual reality research is specifically geared towards realistically imitating real-world phenomena $[45,46]$.

Other authors, however, argue that relatively simplified and idealized representations are useful because they extract the basic principle on which a situation is based $[47,48]$. According to this line of reasoning, this type of representation thus lends itself more easily to application in simulations of other phenomena governed by the same principle [43].

Previous research has indicated the benefits of idealization over concreteness in enabling users to understand the underlying abstract physical principles. It is true that, if we remove all the details and focus on the core basic physical phenomenon, we can make an effective transfer of an abstract phenomenon to other scientific disciplines [43].

In spite of the obvious potential of simulations as learning tools, the results of previous studies examining their educational effectiveness have been inconsistent. It is possible, however, that the increased sophistication and realism of current models might yield different results if those studies were to be repeated. The ineffectiveness of a computer simulation may not be the result of poor design. Steinberg [33] argues that the impact of a simulation depends on the details of the program and how it is implemented. Brant et al. [49] attribute the ineffectiveness of simulations to the inappropriate instructional roles for which they are used in the teaching process and argue that their effectiveness may depend upon when they are administered within an instructional sequence. These works suggest that computer simulations may lead to overdependence on the results of the simulation, inhibiting students' ability to think independently and form hypotheses and logical deductions. For instance, Steinberg states that computer simulations that quickly and transparently deliver exact answers can encourage passive learning among students of science.

Another problem often encountered in working with simulations is the accuracy of the graphical components. As indicated by Chang et al. [4], this accuracy should be increased to prevent problems for students with less capacity for abstraction.

\section{Educational setting}

In a schematic simulation, students are faced with abstract entities that at times lack any real-world connection. Focusing on the field of optics, our mission in this work was to show the students what the abstract phenomenon looks like in reality.

Students of all academic levels misinterpret the behavior of light and the formation of images by mirrors and lenses $[6,18,50-53]$. During our prior teaching experience we found that, even after our students had been taught about the theory of image formation by lenses and mirrors and the presence of aberrations, most of them were unable to connect the images with real-world phenomena. This inability remained even after detailed instruction in the formation of images (our students had already completed their study of geometrical optics and they had, in principle, all the knowledge necessary to solve simple problems).

This may be partly explained by the fact that their textbooks that use the ray-tracing approach stress the central light rays (relative to the optical axis) at the expense of peripheral rays [51]. Ray tracing is, of course, a powerful tool with which to describe and explain image formation in geometrical optics [18,54]. It is useful for both locating the position of an image relative to a lens or mirror and determining the image's size relative to the size of the object. However, ray tracing is sometimes so heavily emphasized that the more basic questions of how and why images are formed are neglected $[18,19,42]$.

Our main objective in this work was to help fill in the gaps in the students' understanding by providing them with the means to conduct real-life observation of a phenomenon they had studied in a schematic computer simulation. Our work is designed to endow these overly schematic classical simulations with a far higher level of realism so as to minimize the difficulties that students with low levels of abstract reasoning often encounter in working with classic simulations.

In the present study, two virtual environments were constructed. One of them is a schematic environment 
implemented in JAVA, in which the students can interact in real time with the physical phenomenon being modeled. The other is based on the first JAVA environment, but the visual output of the JAVA simulation is modified with the ray-tracing program POV-Ray [55]. This program transforms the schematic JAVA graphical user interface into a photorealistic visual output similar to that observed in a real laboratory. We have termed this combination of the real-time interactivity of the schematic simulation in JAVA with the output of photorealistic images "hyper-realism."

Our hyper-realistic virtual environment allows students to visualize and interact with a simulated phenomenon as they would experience it in the real laboratory. As an example of this type of simulation, we shall present a hyper-realistic virtual model for the case study of optical aberrations. Its purposes are twofold. First, it was designed to serve as an educational resource to help the student to better understand the functioning of optical systems in a way that is unavailable in the traditional laboratory. Second, it was intended to complement observations made in real systems. Computer simulations can be used to promote conceptual change and to help students assimilate the concepts involved in the phenomenon. Applying this theory of learning to our simulations, the new concept of photorealism in computer simulation allows us to pose the following research question: Will the hyper-realistic simulations of optical systems be effective in promoting the students' learning and assimilation of the physical concepts involved in the formation of images?

Figure 1 shows one of these hyper-realistic simulations.
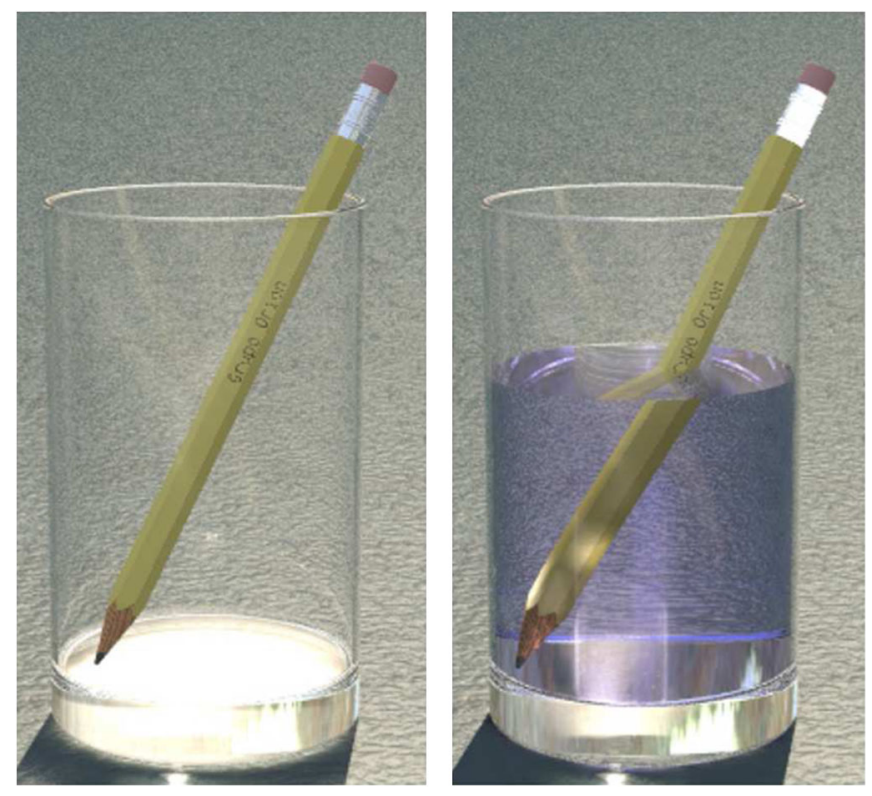

FIG. 1 (color online). Hyper-realistic simulation of a pencil in an empty glass (left). Hyper-realistic simulation of a pencil partially submerged in water $(n=1.33)$ seen from the air $(n=1)$ (right).

\section{METHODS}

\section{A. Hypotheses}

The experiment described in the present work was designed to determine the veracity of the following working hypotheses.

$\mathrm{H} 1$ : The learning achievements of the students using hyper-realistic virtual environments are significantly greater than those of the students working with traditional simulation technology and greater even than those of students working in a traditional laboratory.

The null hypothesis was therefore:

H0: The learning achievements of students using hyperrealistic virtual environments are not significantly greater than those of students working with traditional simulation technology or in a traditional laboratory.

\section{B. Sample}

The experiment was carried out during the 2009-2010 academic year. The study participants were 123 junior and senior students pursuing degrees in several scientific disciplines at the College of Science in the University of Extremadura (Spain). These students were divided into three groups that were homogeneous and equivalent in terms of their degree courses, capabilities, discipline, and academic performance in their previous lecture courses.

The first group was designated the "Hyper-realistic Experimental Group" (Hyp.E.G.) and was composed of 41 students. This group used a hyper-realistic virtual laboratory to complete the practical classes on the formation of images and optical aberrations.

The second group was designated the "Schematic Control Group" (Schem.C.G.) and was composed of 41 students. This group used computer schematic simulations to study the formation of images and optical aberrations.

The third group was designated the "Traditional Control Group" (Trad.C.G.) and was also composed of 41 students. This group used the traditional laboratory, with real optics apparatus, to study the formation of images and optical aberrations.

\section{Experimental design}

The research design was quasiexperimental, with a posttest and two control groups. The independent variable was the method used in the learning process, i.e., the use of either the virtual environment based on hyper-realistic computer simulations (teaching method for the Hyp.E.G.), the virtual environment based on traditional schematic simulations (teaching method for the Schem.C.G.), or the traditional laboratory using real optics systems (teaching method for the Trad.C.G.). The dependent variable was the learning achieved by the students.

Clearly, the differences in learning achieved by each group, which the hypotheses ( $\mathrm{H} 1$ or $\mathrm{H} 0)$ presume to be 
the result of differences in teaching method, may also be contaminated by other variables. The learning achievements may depend not only on how effective each teaching method is, but also on, for instance, possible differences between the three groups of students in terms of their prior knowledge of the topic.

To minimize the influence of this potential interference variable, we chose a topic of a sufficiently high level that none of the students had studied it previously. Regardless of the group to which they were assigned, none of the students selected as subjects for the study had prior knowledge of paraxial optics and aberrations. We determined this using a test of prior knowledge, in which the students were unable to define these concepts or answer questions similar to those found in the post-test. Most of the students had difficulty explaining why and where the images were formed. Furthermore, the homogeneity of the groups allowed us to assume a common baseline of knowledge.

At the beginning of the study unit, students were asked to build a concept map [56] associating the most significant concepts related to image formation in lenses and mirrors.
None of the propositions created by the students in their maps included the concepts of aberration or sharpness of an image. However, these concepts were included by the students in the concept maps they prepared as homework at the end of the unit.

Depending on their group membership, the students received training on handling either the virtual environment simulations (Hyp.E.G. and Schem.C.G.) or the real apparatus in the laboratory (Trad.C.G.). In order to ensure that the three groups received the same initial preparatory training, the laboratory sessions were preceded by a common theoretical class on the formation of optical images. The three groups performed the same practicals in four 3 -hour sessions in their respective laboratories. The practicals were carried out in small groups of two or three students. During these sessions, the students were provided with the scripts of the practical tasks that they were expected to perform. These scripts specified the objective and procedures for every practical, as adapted to their learning environments. By way of example, an excerpt of these scripts is provided in Fig. 2.
IMAGE FORMATION BY A DIVERGING LENS

OBJETIVES:

1. Simulate the physical behavior of a diverging lens.

2. Study the characteristics of the image formed by a diverging lens.

3. Analyze the behavior of the lens in paraxial area and beyond.

4. Study spherical geometric aberration.

5. Analyze how it influences the width of the lens the size of the aberration. OPERATING METHOD:

Consider the lens represented when initializing the simulation.

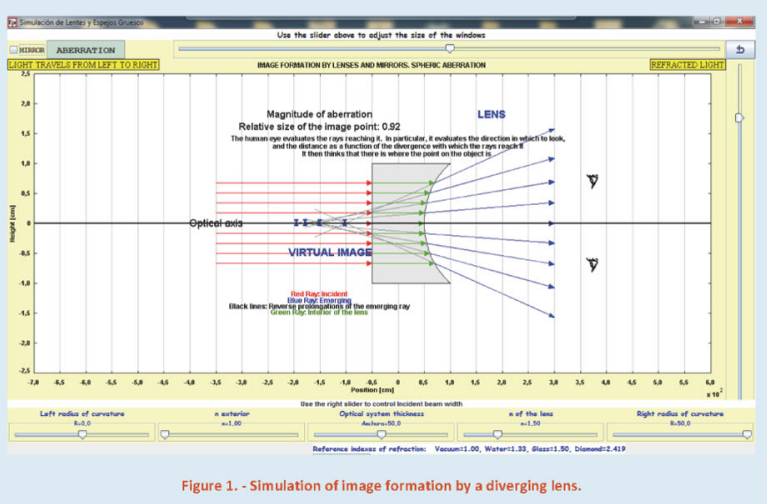

Observe the path of incident and refracted rays:

- Do they intersect?

- Do their extensions intersect?

- Do they intersect at a single point?

- What happens to the refracted rays that are located closer to the edge of the lens? Do they converge or diverge?

- What happens to the refracted rays located closer to the optical axis?

- In what position is the image formed

- Is the image real or virtual? Why?

- Do you see any kind of aberration? Which one?

- How wide (in the $X$ axis) is the observed aberration?

- How could we avoid this aberration?

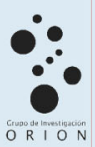

Move the right slider to regulate the width of the incident beam in the system without changing the other parameters and observe how the amplitude of the aberration changes and how the image points are coming closer.
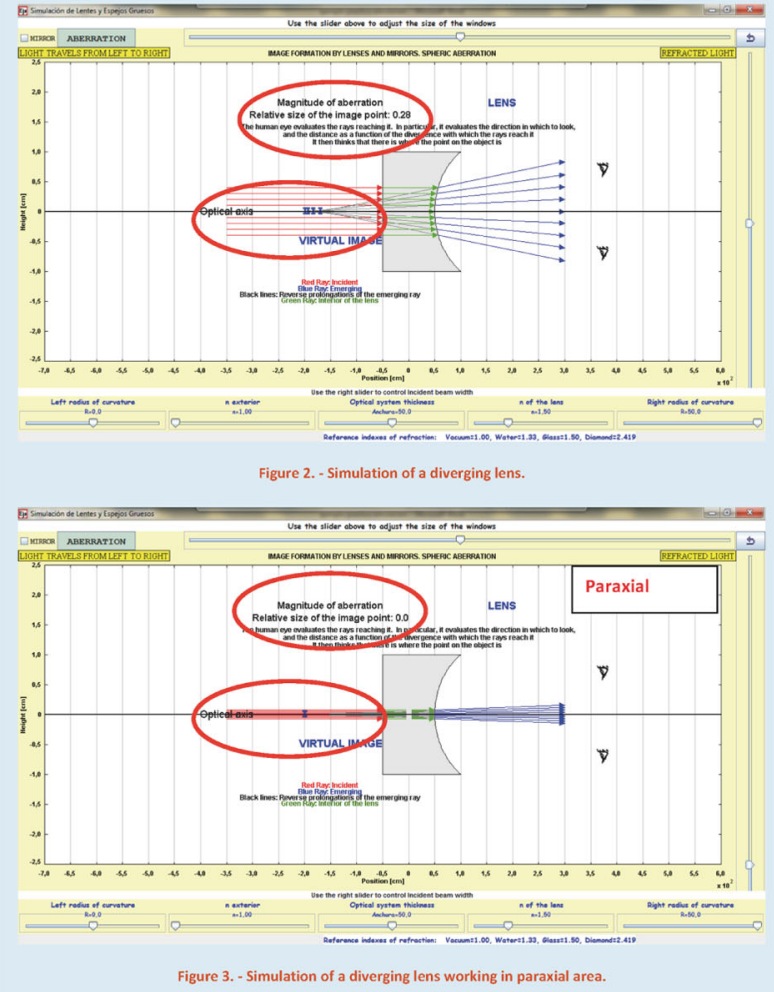

What happens if you move the right slider decreasing the width of the incident beam? In this case, would you modify the answers to the questions above?

Next we will work with other kind of diverging lens: a biconcave lens

FIG. 2 (color online). Excerpt of the scripts provided to the students. 


\section{Educational objective}

The common educational objective of the three laboratories (the hyper-realistic virtual laboratory, the schematic virtual environment, and the real laboratory) was to enable the students to observe the behavior of a beam of light incident on a lens or a mirror. The laboratories were designed to introduce students to cases occurring both within and outside of the paraxial zone and enable them to understand why the images were observed at particular positions. The paradigm of geometrical optics is based on the study of centered optical systems, most often those with surfaces of revolution. This paradigm uses the paraxial approximation, which ensures stigmatic correspondence between each object point and its corresponding image point. This approximation will only hold if the angle subtended by any light ray (the idealization of an infinitesimal beam of light commonly used in this paradigm), with respect to the normal of the surface of the optical element on which it is incident, is small. However, in real optical systems, as this angle increases, the rays from a given object point no longer cross at a single image point but instead form a circle of a certain diameter, called the circle of confusion. Since these disks overlap with each other, there is a loss of image sharpness, with the blurriness increasing with disk size. This phenomenon is known as nonchromatic, or Seidel, optical aberration. The example chosen for simulation was the formation of the image of a single object point at infinity in a simple optical system.

Although there are many available online programs that simulate the phenomena of geometrical optics, most of them are valid only for the paraxial zone and therefore are useful only for illustrating the basic principles $[18,19,57,58]$. They do not, however, allow the student to see the difference between how images are formed within and outside of the paraxial zone. The difference between the real behavior of optical systems and the theoretical behavior depicted by the paraxial approximation often results in students having problems properly understanding optical phenomena. Our hyper-realistic virtual environment allows students to study the images formed using both ideal and real-world optical models.

\section{E. Description of the hyper-realistic virtual environment}

\section{Creation of the interactive schematic JAVA environment}

The schematic and interactive environment of our simulation was developed using the program EJS (Easy Java Simulations) [59]. This software platform, developed for the Open Source Physics Project [60-62], allows the user to create scientific simulations in JAVA. We chose this program because it greatly facilitates the creation of scientific applets. Its numerous tools facilitate the description of the model of the simulated phenomenon and, by

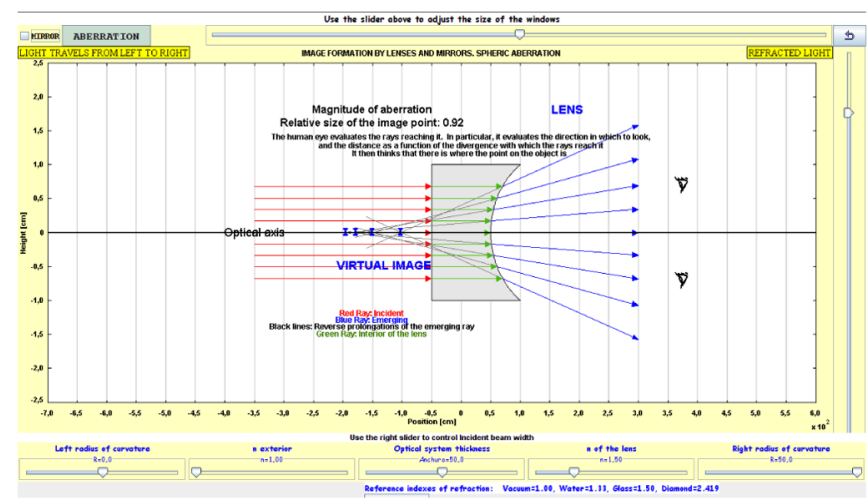

FIG. 3 (color online). View of the schematic JAVA environment developed to illustrate image formation by lenses and mirrors.

simplifying the purely programming aspects, allow one to focus on the content of the simulation. We used this program to specify the model for the simulated system and to construct a display that continuously shows the model's status and responds interactively to the user's actions. The student does not need to input or modify the equations in the mathematical model since these were coded into the program when the simulation was created. Instead, the student can focus on observing the results of varying the parameters. The applet is based on geometrical optics ray tracing, but it allows for the possibility of working with an incident light beam, either inside or outside of the paraxial zone. We selected the following parameters for student modification and interaction with the simulation: the type and position of the optical system, the refractive index of the external medium, the width of the incident beam, the radius of curvature (for mirrors), and the refractive index and the two radii of curvature (for lenses).

Figure 3 is a screen shot of the resulting schematic JAVA environment.

The mathematical model used in the simulation is based on the Seidel formulas for rays incident on an optical system that has a non-negligible aperture, thus leading to spherical aberration. Spherical aberration refers to a situation wherein rays emanate from an object point located at a far distance on the optical axis, so that they are incident parallel to that axis and it includes rays that are appreciably separated from it. If the lens is not ideal, then the rays passing through its center and those passing through its periphery cross at different places, resulting in an image depicting a circle of confusion instead of the single sharp point produced when working in paraxial optics.

\section{Creation of a photorealistic visual output using POV-Ray}

To achieve a visually photorealistic output, we chose the program POV-Ray (persistence of vision raytracer) [55], an open-source raytracer. Our choice of this program was 


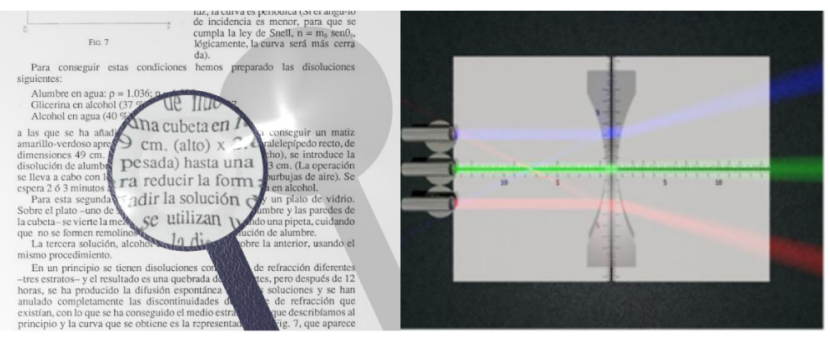

FIG. 4 (color online). Left: Hyper-realistic simulation of the behavior of a converging lens over a printed text. Right: Hyperrealistic simulation of the behavior of the effects of three incident rays parallel to the optical axis of a diverging lens.

determined by our need for a technique capable of faithfully imitating the optical system in a manner that was consistent with the theoretical models involved. POV-Ray uses a geometrical optics-based ray-tracing technique that synthesizes images with great realism [63]. It models the light's path by following the rays as they interact with optical surfaces, yielding accurate simulations of phenomena, such as reflection, refraction, and shadows. These simulations emerge as a natural result of the combined use of the ray-tracing algorithm and a specific Monte Carlo algorithm for the synthesis of threedimensional images with perspective. The program implements additional algorithms, such as photon mapping, which add realism to the overall illumination of the scene. Furthermore, the program is open source and available for almost all computer platforms. It allows the user to represent objects internally with mathematical functions using a scene description language. This is a major advantage; the user then only has to be concerned with the geometric description of the optical system (light source, object, and observer) because all of the underlying optics (Seidel formulas, Snell's law, the Fresnel equations, etc.) already form part of the program's source code.

Within the schematic interactive JAVA environment, the user of our simulations can modify the position of the optical system, the radii of the curvature, the thickness of the lens and its refractive index, the refractive index of the external medium, and the width of the incident light beam. All these data, which are purely numerical, are exported to a file that describes the optical system geometrically. This file is interpreted by POV-Ray, which renders a photorealistic image of the scene that has been generated in JAVA.

Figure 4 depicts an example of two hyper-realistic simulations rendered by POV-Ray.

\section{F. Evaluation instruments}

To quantify the learning achieved by the students in the three groups (Hyp.E.G., Schem.C.G., and Trad.C.G.), we designed a test to serve as the evaluation instrument. Initially, we drafted a 25 -question test, which we gave to a pilot group of 20 students who were not going to

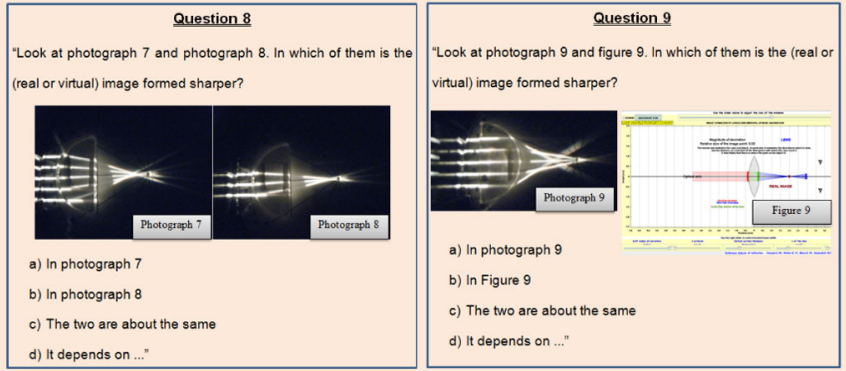

FIG. 5 (color online). Two of the questions of the test. The full test can be seen in Ref. [68].

participate in the study. The results were processed to remove those questions that did not demonstrate a sufficient discriminatory index. The final test, then, consisted of 20 closed-response items, each with 4 possible answers, of which only one was true. To determine the reliability of this test, the KR-20 coefficient was calculated [64]. The value found was 0.62 , which we considered moderate [65].

This test was given to each group as a post-test at the end of their study of this unit. To minimize the potential inference of the variable described by Dancy and Beichner [66] (whereby a student's verbal skills may influence his or her test results), the items in our test were presented together with images.

By way of example, Fig. 5 provides two of the test questions.

As shown in Fig. 5, the test questions were posed to allow the students to compare the images formed by the lenses in two different situations. This enabled us to assess whether or not they were able to relate the developed concept of geometric aberration with the sharpness of the image formed on the optical system. The photographs and images used in the test items were equally distributed to ensure that the experimental and control groups were exposed to equal pretest conditions. That is, there were an equal number of questions using each visual tool (photographs, schematic images, and photorealistic images) [67]. The full test can be accessed from Ref. [68].

\section{RESULTS AND DISCUSSION}

\section{A. Realism of the hyper-realistic virtual environment}

In order to indicate the realism achieved by the hyperrealistic virtual environment, we will now provide some of the simulation's graphical outputs, along with equivalent photographs taken by the Trad.C.G. students during their practicals in the real-life optics laboratory.

As shown in Fig. 6, both the JAVA applet and the hyperrealistic simulation faithfully reproduce the behavior of light passing through the optical system, but the view generated by the hyper-realistic simulation is much closer to reality than the view generated by the JAVA applet. 


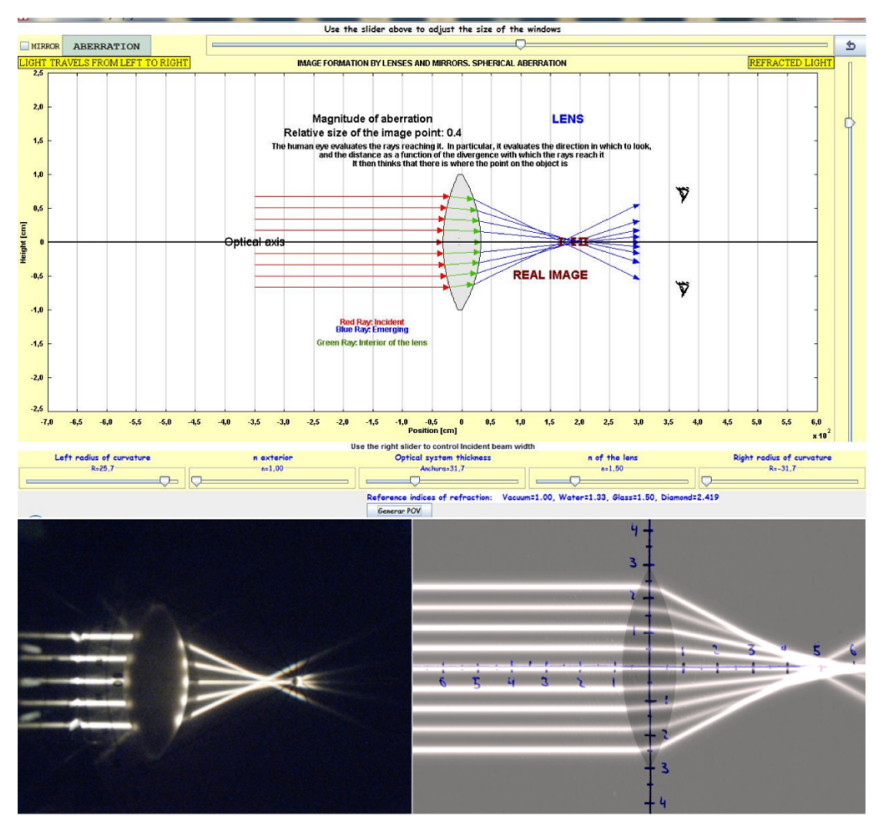

FIG. 6 (color online). Top: Virtual laboratory: View generated by the JAVA simulation of spherical aberration in a section of a biconvex lens. Bottom left: Photograph of a section of a biconvex lens working outside the paraxial zone. Bottom right: Hyperrealistic simulation of the same optical system.

\section{B. Comparison of the learning achieved in the hyper-realistic virtual environment with the learning achieved in the schematic JAVA environment and the real laboratory}

We analyzed the data obtained from the evaluation tests using a statistical package. This analysis allowed us to evaluate and compare the learning achieved by the students in each group. Figure 7 shows the histogram of the percentages of correct answers given by the Hyp.E.G., Schem.C.G., and Trad.C.G. students.

Figure 7 demonstrates that the mean scores of the three groups differed: the mean test score for Hyp.E.G. was $(76.46 \pm 2.27) \%$, the mean test score for Schem.C.G. was $(70,36 \pm 1,94) \%$, and the mean test score for Trad.C.G. was $(66.95 \pm 2.25) \%$. We will now examine these differences in detail. To check whether this difference reached the level of significance $(5 \%$, or $p<0.05)$ and to test the validity of the working hypothesis, we subjected the test scores to an analysis of variance (ANOVA). The assumptions of randomness, normal distribution, and homoscedasticity of the sample data were tested and, since these three preconditions were satisfied, we considered the use of the ANOVA justified. The results are presented in Table I.

Since the $p$ value (0.008) was markedly less than 0.05 , we concluded that, at the chosen $95 \%$ confidence level, the difference between the mean scores of the three groups was significant, i.e., the differences were not due to chance but rather were a result of the respective teaching methods used for each group. Thus, at least one of the teaching environments enables significantly different learning results.

In order to assess separately the results, we applied a student's $t$-test to independent samples. The results are given in Table II. The table (top) shows that the mean score for Hyp.E.G. was 9.5 percentage points greater than the mean score for Trad.C.G. and the $p$ value was less than 0.05 . The middle part of the table shows that the mean score for Hyp.E.G. was 6.1 percentage points greater than the mean score for Schem.C.G. and the $p$ value was less than 0.05 . The bottom part of the table shows that the mean score for Schem.C.G. was 3.4 percentage points greater than the mean score for Trad.C.G. and the $p$ value was greater than 0.05 . These results demonstrate that learning based on hyper-realistic virtual environments was significantly more effective than learning attained through traditional schematic simulations and even more effective than learning attained in the real laboratory. However, there was no statistically significant difference between the learning attained through the traditional schematic simulation and the learning attained in the real laboratory.

The two columns on the far right-hand side of Table II show Cohen's $d$ and the effect size coefficients of the results. As shown, the effect size of the schematic simulations versus actual laboratory is $r=0.128$. However, the effect size of the hyper-realistic simulations versus the schematic simulations is $r=0.222$. The effect size becomes even greater if we directly compare the hyper-realistic simulations to the traditional laboratory $(r=0.316)$.

\section{End-user evaluation}

To determine the end-users' evaluation of the hyperrealistic simulations, we used two evaluation instruments, one targeting the teachers and one targeting the students.

\section{Assessment by the teachers}

We prepared a 4-item, 5-level Likert scale [69] questionnaire addressed to the 10 expert teachers giving the optics classes. The items were as follows. Q1: The simulation is successful in motivating the student. Q2: The simulation is effective in developing skills. Q3: The method is applicable to other physical phenomena. Q4: The simulation is effective for teaching conceptual content. Four additional open-ended items were included to allow the teachers to provide feedback and comments on possible improvements. The quantitative data obtained from this questionnaire were analyzed using a statistical package. Figure 8 shows the percentages corresponding to each Likert scale item in the teachers' responses.

Figure 8 demonstrates that the teachers' overall assessment of the hyper-realistic simulation was very positive. It is worth noting that $\mathrm{Q} 4$ was the most highly rated item according to the Likert scale. This item assesses the effectiveness of the simulation for learning conceptual 

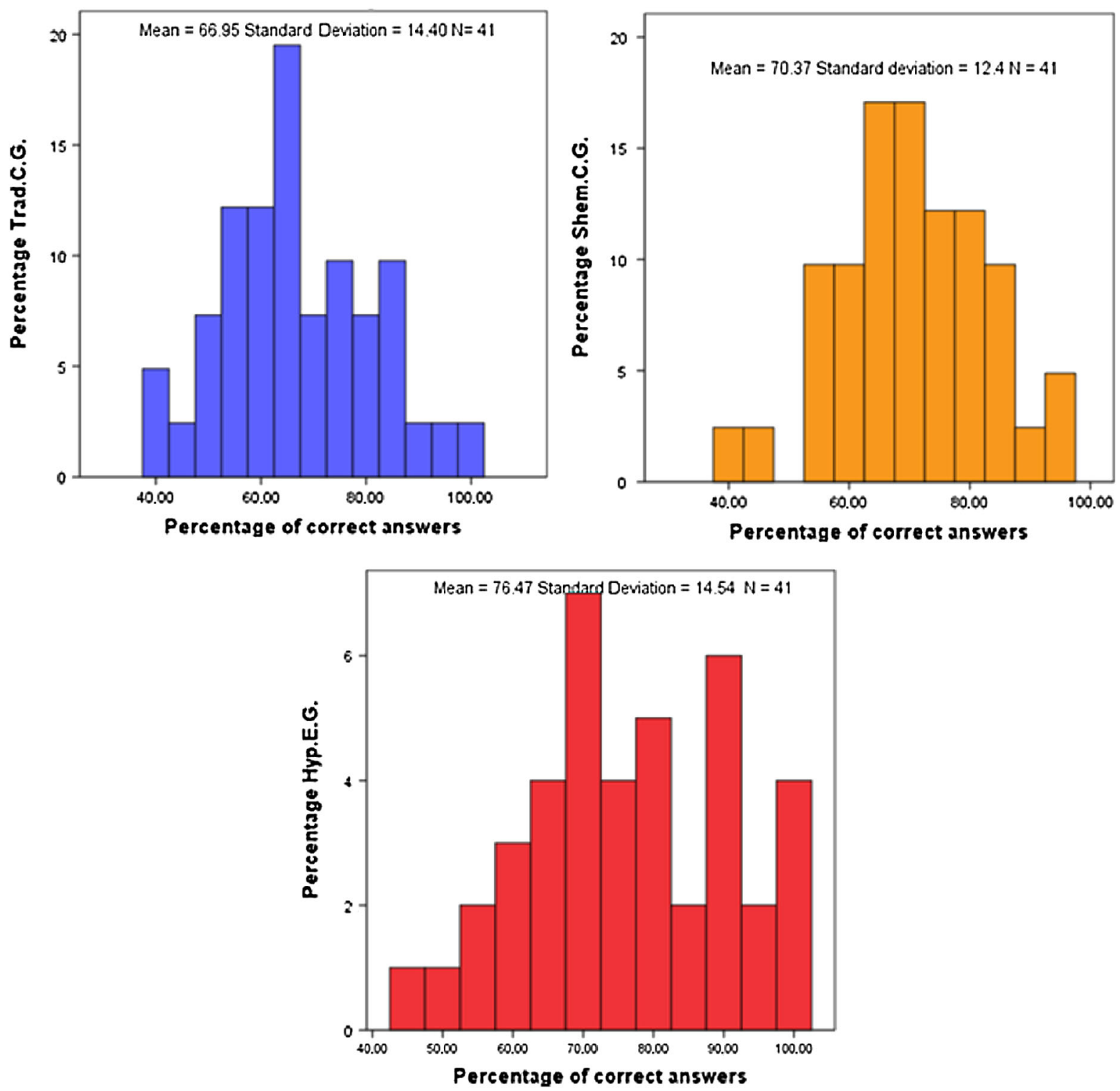

FIG. 7 (color online). Histogram of the percentage of correct answers for the Trad.C.G. (top left), the Schem.C.G. (top right), and the Hyp.E.G. (bottom) groups.

contents. Sixty percent of the teachers surveyed, who were all experts in the field, strongly agreed with this statement and $40 \%$ agreed; there were no neutral or negative responses for this item.

\section{Assessment by the students}

Another questionnaire was addressed to the 41 Hyp.E.G. students who had used the hyper-realistic virtual environ-

TABLE I. ANOVA of the variable "learning."

\begin{tabular}{lcrccc}
\hline \hline ANOVA & Sum of squares & d.o.f. & Mean square & \multicolumn{1}{c}{$F$} & $\sigma$ \\
\hline Between groups & 1904.065 & 2 & 952.033 & $4.983 \mathbf{0 . 0 0 8}$ \\
Within groups & 22925.610 & 120 & 191.047 & & \\
Total & 24829.675 & 122 & & & \\
\hline \hline
\end{tabular}

ment. The purpose of this questionnaire was to evaluate two attributes of the virtual environment: its "technical aspects" and its "educational aspects." We first prepared a trial 15-item, 5-level Likert scale questionnaire, which we presented to a pilot group. Three of the items were then rejected on the basis of statistical criteria. Of the 12 closed questions that we ultimately accepted, six referred to technical aspects of the simulation and six to educational aspects. Three open-ended items were added to enable the students to include their comments. These items were used to study their suggestions for possible improvements. The questionnaire is shown in Fig. 9. The response data were subjected to a statistical analysis. The results are presented in Fig. 10. As was the case with the teachers' responses, the students' overall assessment of the virtual hyper-realistic simulation was very positive. 
TABLE II. Student's $t$-test for the equality of means for independent samples $(p<0.05)$, with Cohen's $d$ and effect size coefficient r. Hyp.E.G. versus Trad.C.G. (top), Hyp.E.G. versus Schem.C.G. (middle), Schem.C.G. versus Trad.C.G. (bottom). CG1, control group 1; CG2, control group 2; GE, experimental group.

\begin{tabular}{lcccccc}
\hline \hline$t$ & d.o.f. & $\sigma$ (bilateral) & Difference of means GE versus GC2 & Standard error of the difference & $d$ & $r$ \\
2.98 & 80 & 0.004 & 9.51 & 3.20 & 0.67 & 0.316 \\
$t$ & d.o.f. & $\sigma$ (bilateral) & Difference of means GE versus GC1 & Standard error of the difference & $d$ & $r$ \\
2.04 & 80 & 0.045 & 6.10 & 2.99 & 0.46 & 0.222 \\
$t$ & d.o.f. & $\sigma$ (bilateral) & Difference of means GC1 versus GC2 & Standard error of the difference & $d$ & $r$ \\
1.15 & 80 & 0.254 & 3.41 & 2.97 & 0.26 & 0.128 \\
\hline \hline
\end{tabular}

\section{CONCLUSIONS}

In this study, we have implemented a new type of computer simulation, which we term hyper-realistic, that represents reality with far greater realism than do commonly used simulation programs.

We have described the design, application, and evaluation of a hyper-realistic simulation of the phenomenon of image formation in a mirror or lens for both an ideal (stigmatic) system and one including optical aberrations.

The results of this application of our hyper-realistic virtual environment demonstrated that the students using it achieved greater learning accomplishments than those who used traditional simulations or a real laboratory. We were thus able to confirm our working hypothesis: "The learning achieved by students using hyper-realistic virtual environments is significantly greater than the learning achieved by students working with traditional simulation and even greater than the learning achieved by students working in a traditional laboratory." Indeed, the hyperrealistic virtual environment resulted in better assimilation of concepts; the scores of the Hyp.E.G. students were 6.1 percentage points higher than those of the Schem.C.G. and

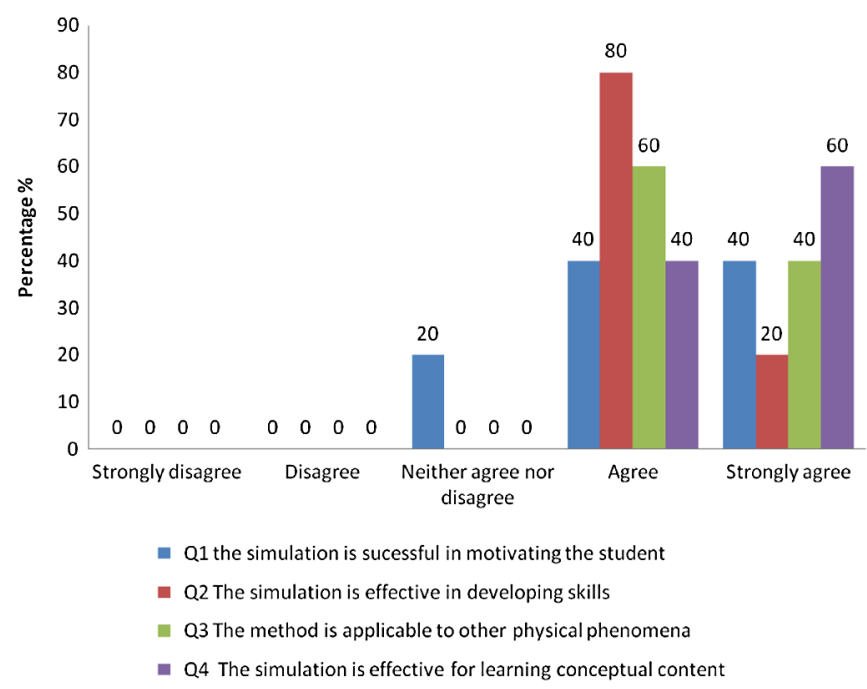

FIG. 8 (color online). Graphical representation of the teachers' assessment.
9.5 percentage points higher than those of the Trad.C.G. students.

Previous studies indicate that a higher degree of learning is achieved through schematic simulations than in a traditional laboratory, although this increase is not always statistically significant. Our results using the schematic simulations corroborate these previous studies; we found that learning achievement was 3.4 percentage points higher for schematic simulations than for traditional laboratory practices. Although this difference is not statistically significant in this case study, it is educationally significant, as we have also observed in our teaching over the past few years. This supports our belief that computer simulations should mainly be used as a complementary tool to reinforce traditional laboratory learning and should only replace the traditional laboratory if the latter is unavailable, as is the case in e-learning.

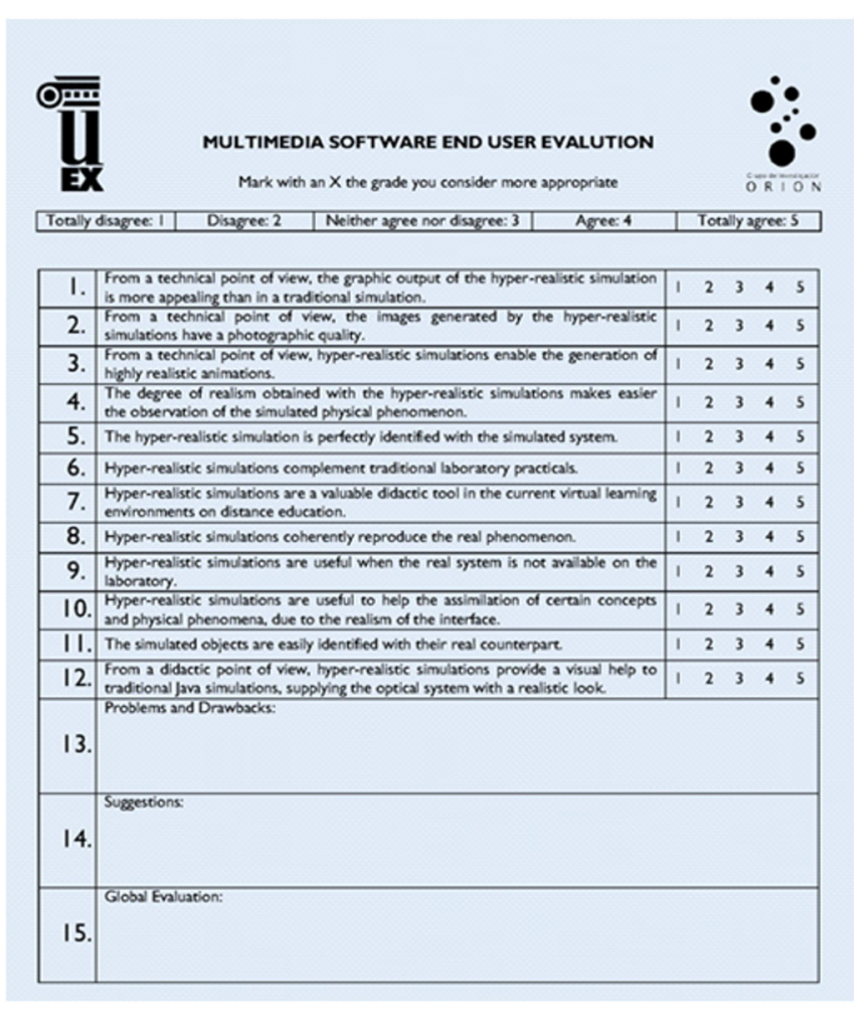

FIG. 9 (color online). Questionnaire used on the students' assessment. 


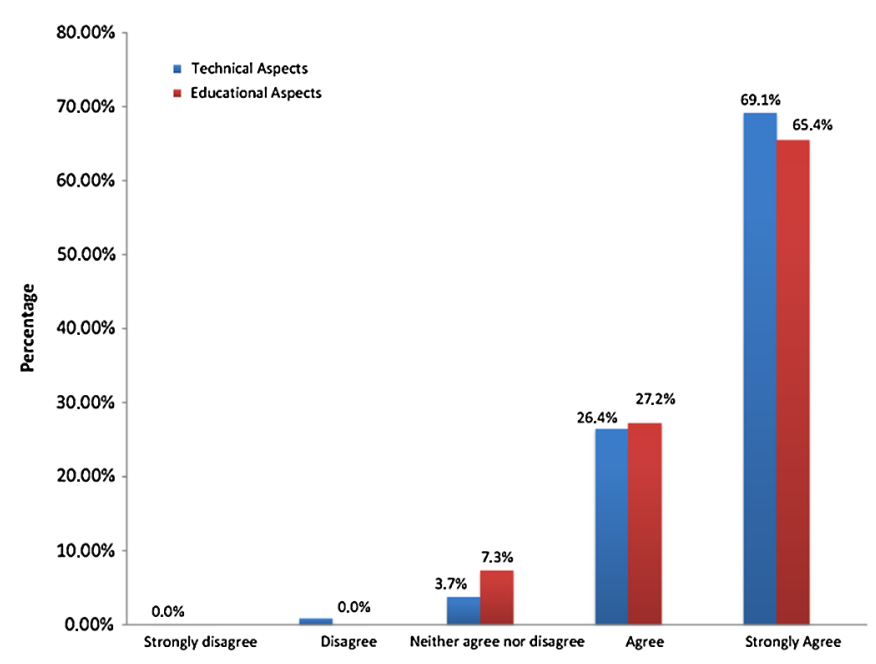

FIG. 10 (color online). Graphical representation of the students' assessment.

However, the main innovation of our study lies in its demonstration of the effectiveness of hyper-realistic simulations. In this case, the results were both statistically and educationally significant because they demonstrated that hyper-realistic simulations produced greater learning achievement than did traditional schematic simulations or the physical laboratory.

The hyper-realistic virtual environment that we created redressed some of the weaknesses found in commonly used computer simulations, such as their simplicity and lack of realism of the graphical environment that the student sees. The result was that the students using our simulations attained, on average, greater learning achievement than those who used traditional simulations or the physical laboratory. A possible reason for this may be that, in the virtual hyper-realistic environment, what the student receives is previously filtered information, allowing his or her attention to be focused on the most relevant aspects of the experiment. In addition, the simulation's degree of concreteness can be altered from schematic to hyperrealistic. This allows the students to explore the practicals at different levels of concreteness, which makes it easier for them to test experimentally the provisional hypothesis they have proposed in constructing their interpretation of the observed phenomenon. They can thus immediately reject incorrect interpretations and reinforce their understanding of the correct ones.

These simulations give the students the opportunity to carry out optical practicals at any time and from any place. This learning method thus eliminates the problems that often arise in the study of inherently practical subjects, whereby the lack of material resources outside of the laboratory limits the students' work to mere theoretical studies.
Appropriately designed hyper-realistic computer simulations are very effective teaching tools in certain educational contexts, such as e-learning teaching platforms. We expect that these results can be generalized to other content areas, provided that the simulated phenomena present observable behaviors to the naked eye.

However, there are some situations in which the use of hyper-realistic simulations does not enhance the schematic JAVA simulations. We must keep in mind that the educational effectiveness of a hyper-realistic simulation lies in its ability to address the discrepancy between simulated study and practical observation In this regard, we must distinguish between the simulations that simulate the result and simulations that simulate the development of the experience. Thus, schematic JAVA simulations are useful in simulating the results and representing the values of the variables involved in the mathematical model. For example, in creating simulations on the law of radioactive decay or chemical kinetics, the inclusion of hyper-realistic outputs of the model would not enhance the schematic simulation. Such simulations are effective when the details are removed and we can focus schematically on the underlying basis of the physical phenomenon, thus enabling an effective transfer of the abstract phenomenon being simulated to other scientific fields. In other words, schematic JAVA simulations sometimes present students with abstract entities that lack connection to the visual appearance of the phenomenon in the real world but may be useful in some fields of physics.

Nevertheless, we believe that in certain fields of physics, such as optics, our area of specialization, simulations that simulate the development of the experience, that is, provide a realistic visual output of the simulated phenomenon, are highly effective teaching tools. These simulations show students how an abstract phenomenon simulated schematically in JAVA operates in reality, creating a hyper-realistic simulation of the phenomenon in its entirety.

That is, hyper-realistic simulations are especially useful for the representation of optical phenomena, because the phenomenon being simulated is what is seen, so that the student identifies what he sees happening in the simulated model with what he sees happening in reality. We expect that the results will be applicable to other student groups as well.

\section{ACKNOWLEDGMENTS}

The authors wish to thank the Regional Government of Extremadura for its support through Grant No. GR10102, partially funded by the European Regional Development Fund. 
[1] T. Martin and A. Serrano, The role of new technologies in the learning process: Moodle as a teaching tool in physics, Comput. Educ. 52, 35 (2009).

[2] R. Kenny, M. Bullen, and J. Loftus, A pilot study of problem formulation and resolution in an online problem-based learning course, Int. Rev. Res. Open Dist. Learn. 7, 1 (2006) [http://www.irrodl.org/index.php/irrodl/ article/view/248].

[3] I. Halloun, Schematic modeling for meaningful understanding of physics, J. Res. Sci. Teach. 33, 1019 (1996).

[4] K. E. Chang, Y. L. Chen, H. Y. Lin, and Y. T. Sung, Effects of learning support in simulation-based physics learning, Comput. Educ. 51, 1486 (2008).

[5] D. R. Sokoloff and R. K. Thornton, Using interactive lecture demonstrations to create an active learning environment, Phys. Teach. 35, 340 (1997).

[6] J. A. Bryan and S. W. Slough, Converging lens simulation design and image predictions, Phys. Educ. 44, 264 (2009).

[7] T. L. Naps et al., Exploring the role of visualization and engagement in computer science education, SIGCSE Bull. 35, 131 (2003).

[8] E. Lewis, J.L. Stern, and M. C. Linn, The effect of computer simulations on introductory thermodynamics understanding, Educ. Technol. 33, 45 (1993).

[9] M. Ronen and M. Eliahu, Simulation-a bridge between theory and reality: The case of electric circuits, J. Comput. Assist. Learn. 16, 14 (2000).

[10] N. D. Finkelstein, W. K. Adams, C. J. Keller, P. B. Kohl, K. K. Perkins, N. S. Podolefsky, and S. Reid, When learning about the real world is better done virtually, Phys. Rev. ST Phys. Educ. Res. 1, 010103 (2005).

[11] R. Trumper, The physics laboratory-A historical overview and future perspectives, Sci. Educ. 12, 645 (2003).

[12] Z. Zacharia and O. Roger Anderson, The effects of an interactive computer-based simulation prior to performing a laboratory inquiry-based experiment on students' conceptual understanding of physics, Am. J. Phys. 71, 618 (2003).

[13] R. Landau, Computational physics: A better model for physics education? Comput. Sci. Eng. 8, 22 (2006).

[14] F. K. Hwang, Virtual Physics Laboratory, http://phy .ntnu.edu.tw/ntnujava/.

[15] P. Tao and R. Gunstone, The process of conceptual change in force and motion during computer-supported physics instruction, J. Res. Sci. Teach. 36, 859 (1999).

[16] P. Gorsky and M. Finegold, Using computer simulations to restructure student's conceptions of force, J. Comput. Math. Sci. Teach. 11, 163 (1992).

[17] F. Goldberg, Constructing physics understanding in a computer-supported learning environment, AIP Conf. Proc. 399, 903 (1997).

[18] B. S. Eylon, M. Ronen, and U. Ganiel, Computer simulations as tools for teaching and learning: Using a simulation environment in optics, J. Sci. Educ. Technol. 5, 93 (1996).

[19] P. Tao, Developing understanding of image formation by lenses through collaborative learning mediated by multimedia computer-assisted learning programs, Int. J. Sci. Educ. 26, 1171 (2004).

[20] Z. Zacharia, Beliefs, attitudes, and intentions of science teachers regarding the educational use of computer simu- lations and inquiry-based experiments in physics, J. Res. Sci. Teach. 40, 792 (2003).

[21] A. Van Heuvelen, Using interactive simulations to enhance conceptual development and problem solving skills, AIP Conf. Proc. 399, 119 (1997).

[22] R. Beichner, L. Bernold, E. Burniston, P. Dail, R. Felder, J. Gastineau, M. Gjersten, and J. Risley, Case study of the physics component of an integrated curriculum, Am. J. Phys. 67, S16 (1999).

[23] J. Snir, C. Smith, and L. Grosslight, Conceptually enhanced simulations: A computer tool for science teaching, in Software Goes to School: Teaching for Understanding with New Technologies, edited by D. N. Perkins, J.L. Schwartz, M.M. West, and M.S. Wiske (Oxford University Press, New York, 1995), p. 106.

[24] S. M. Lea, B. A. Thacker, E. Kim, and K. M. Miller, Computer assisted assessment of student understanding in physics, Comput. Phys. 8, 122 (1994).

[25] W. Hewson, Diagnosis and remediation of an alternate conception of velocity using a microcomputer program, Am. J. Phys. 53, 684 (1985).

[26] F. Goldberg and S. Bendall, Making the invisible visible: A teaching/learning environment that builds on a new view of the physics learner, Am. J. Phys. 63, 978 (1995).

[27] J. J. Kaput, Creating cybernetic and psychological ramps from the concrete to the abstract: Examples from multiplicative structure, in Software Goes to School: Teaching for Understanding with New Technologies (Ref. [23]), p. 130.

[28] H. M. Doerr, Experiment, simulation and analysis: An integrated instructional approach to the concept of force, Int. J. Sci. Educ. 19, 265 (1997).

[29] N. Shin, D. H. Jonassen, and S. McGee, Predictors of wellstructured and ill-structured problem solving in an astronomy simulation, J. Res. Sci. Teach. 40, 6 (2003).

[30] N. D. Finkelstein, K. K. Perkins, W. Adams, P. Kohl, and N. Podolefsky, Can computer simulations replace real equipment in undergraduate laboratories?, AIP Conf. Proc. 790, 101 (2005).

[31] C. Baily and N.D. Finkelstein, Development of quantum perspectives in modern physics, Phys. Rev. ST Phys. Educ. Res. 5, 010106 (2009).

[32] K. Perkins, W. Adams, M. Dubson, N. D. Finkelstein, S. Reid, and C. Wieman, PhET: Interactive simulations for teaching and learning physics, Phys. Teach. 44, 18 (2006).

[33] R. N. Steinberg, Computers in teaching science: To simulate or not to simulate?, Am. J. Phys. 68, S37 (2000).

[34] T. De Jong and W. R. Van Joolingen, Scientific discovery learning with computer simulations of conceptual domains, Rev. Educ. Res. 68, 179 (1998).

[35] K. Cummings, J. Marx, R. Thornton, and D. Kuhl, Evaluating innovations in studio physics, Am. J. Phys. 67, S38 (1999).

[36] A. Aleksandrova and N. Nancheva, Electromagnetism: Interaction of simulation and real lab experiment, Int. J. Inf. Technol. Knowl. 1, 44 (2007).

[37] A. L. Pérez, J. J. Peña, and B. Mahedero, Electronic device of didactic and electrometric interest for the study of $R L C$ circuits, Am. J. Phys. 47, 178 (1979). 
[38] J. L. Calvo, J. J. Peña, and A. L. Pérez, Notes on experiments: Teaching oscillations with a small computer, Phys. Educ. 18, 172 (1983).

[39] M. I. Suero, A. L. Pérez, P. J. Pardo, and F. Vivas, Prismatrón: Simulation of the refraction of light by a prism as a didactic tool, Opt. Pura Apl. 41, 9 (2008) [http://www.sedoptica.es/OPA/Pdfs/270.pdf].

[40] P. J. Pardo, A. L. Pérez, and M. I. Suero, Euro: A new color vision test in the pockets of three hundred million Europeans, Opt. Express 10, 527 (2002) [http://www .opticsinfobase.org/oe/abstract.cfm?URI=oe-10-13-527].

[41] P. J. Pardo, A. L. Pérez, and M. I. Suero, A new colour vision test in a PC-based screening system, Displays 21, 203 (2000).

[42] A. L. Pérez, M.I. Suero, P. J. Pardo, and J. Gil, Misconceptions in optics: Their persistence at university level, J. Sci. Educ. 4, 70 (2003).

[43] R. L. Goldstone and J. Y. Son, The transfer of scientific principles using concrete and idealized simulations, J. Learn. Sci. 14, 69 (2005).

[44] N. DiFonzo, D. A. Hantula, and P. Bordia, Microworlds for experimental research: Having your (control and collection) cake, and realism too, Behav. Res. Meth. Instrum. Comput. 30, 278 (1998).

[45] S. M. Grady, Virtual Reality: Computers Mimic the Physical World, in Facts on File Science Series (Infobase Publishing, New York, 1998).

[46] M. Heim, Virtual Realism (Oxford University Press, New York, 2000).

[47] R. Gianutsos, Driving advisement with the Elemental Driving Simulator (EDS): When less suffices, Behav. Res. Meth. Instrum. Comput. 26, 183 (1994).

[48] R. L. Goldstone and Y. Sakamoto, The transfer of abstract principles governing complex adaptive systems, Cogn. Psychol. 46, 414 (2003).

[49] G. Brant, E. Hooper, and B. Sugrue, Which comes first the simulation or the lecture?, J. Educ. Comput. Res. 7, 469 (1991).

[50] S. Bendall, F. Goldberg, and I. Galili, Prospective elementary teachers' prior knowledge about light, J. Res. Sci. Teach. 30, 1169 (1993).

[51] P. Colin, F. Chauvet, and L. Viennot, Reading images in optics: Students' difficulties and teachers' views, Int. J. Sci. Educ. 24, 313 (2002).

[52] I. Galili and A. Hazan, Learners' knowledge in optics: Interpretation, structure, and analysis, Int. J. Sci. Educ. 22, 57 (2000).

[53] L. Viennot and W. Kaminski, Can we evaluate a critical detail of teaching practice? The case of a type of diagram in understanding optical imaging, Int. J. Sci. Educ. 28, 1867 (2006).

[54] I. Galili, S. Bendall, and F. Goldberg, Effects of prior knowledge and instruction on understanding image formation, J. Res. Sci. Teach. 30, 271 (1993).

[55] Persistence of vision raytracer (POV-Ray), http:// www.povray.org/.

[56] In additional to the post-test, as standard methodology in our teaching practice, we use concept maps. Because the maps are created by our students, we use them to understand their thinking. These maps allow us to assess, from the propositions that the students build, their ability to structure, prioritize, and relate the concepts involved in the issue. The concept map shows us, in a schematic and meaningful form, a graphical depiction of students' knowledge of a particular topic. This, in turn, reflects the students' ways of thinking about the topic of study and enables us to quantize how much knowledge has been acquired as a result of a meaningful learning process. The concept map depicts a series of concepts, organizes them in a hierarchical manner, and conveys the relationships established between them; the map makes explicit the meanings that students have given to each item. These teaching tools reveal instances in which students have conceptualized incorrect relationships, thus enabling us to identify misconceptions. The structure of the map allows the teacher to identify the student's perceptions and misunderstandings.

[57] M. Ronen and B. Eylon, To see or not to see: The eye in geometrical optics-when and how?, Phys. Educ. 28, 52 (1993).

[58] M. Reiner, R. Pea, and D. Shulman, The impact of simulator-based instruction on the diagramming of the interaction of light and matter by introductory physics students, J. Sci. Educ. Technol. 4, 199 (1995).

[59] Easy Java simulations. http://www.um.es/fem/Ejs.

[60] F. Esquembre, Easy Java Simulations: A software tool to create scientific simulations in Java, Comput. Phys. Commun. 156, 199 (2004).

[61] C. A. Jara, F. A. Candelas, F. Torres, S. Dormido, F. Esquembre, and O. Reinoso, Real-time collaboration of virtual laboratories through the internet, Comput. Educ. 52, 126 (2009).

[62] J. Sánchez et al., Easy Java Simulations: An open-source tool to develop interactive virtual laboratories using MATLAB/Simulink, Int. J. Eng. Educ. 21, 789 (2005) [ http://www.ijee.ie/latestissues/Vol21-5/Ijee1685.pdf].

[63] G. Dolling, M. Wegener, S. Linden, and C. Hormann, Photorealistic images of objects in effective negativeindex materials, Opt. Express 14, 1842 (2006).

[64] G.F. Kuder and M.W. Richardson, The theory of the estimation of test reliability, Psychometrika 2, 151 (1937).

[65] J. M. Cortina, What Is coefficient alpha? An examination of theory and applications, J. Appl. Psych. 78, 98 (1993).

[66] M. H. Dancy and R. Beichner, Impact of animation on assessment of conceptual understanding in physics, Phys. Rev. ST Phys. Educ. Res. 2, 1 (2006).

[67] Specifically, the distribution of representations in the test was as follows: three questions with two real representations, three questions with two schematic representations, three questions with two hyper-realistic representations, three questions with a real and a schematic representation, three questions with a real and a hyper-realistic representation, three questions with a schematic and a hyper-realistic representation and two questions with all three types of representations.

[68] http://grupoorion.unex.es/orion/index.php?option=com_ content $\&$ view $=$ article $\& i d=116$

[69] R. Likert, A technique for the measurement of attitudes, Arch. Psychol. 140, 1 (1932). 